\title{
Investigation of Matrix-induced Interferences in Mixed-gas Helium-Argon Inductively Coupled Plasma Mass Spectrometry
}

\author{
Brenda S. Sheppard*, Wei-Lung Shen*, and Joseph A. Caruso \\ Department of Chemistry, University of Cincinnati, Cincinnati, Ohio, USA
}

The effects of various matrix constituents, $\mathrm{Cd}, \mathrm{Co}, \mathrm{Pb}$, and synthetic ocean water, on analyte ion signal were investigated in He-Ar plasma source mass spectrometry. Analyte ion signal suppressions and enhancements were observed in the presence of varying concomitants. The method used for optimizing analyte ion lens signal determined whether suppression or enhancement was encountered. Tuning on a nitric acid standard solution results in a suppressed signal, whereas tuning on the analyte in the presence of the matrix results in signal enhancement, relative to that obtained with no concomitant present. The heavier mass lead concomitant had the greatest effect on the ion signal. Additionally, lighter analyte elements were affected to a greater extent than heavier analytes in the presence of high concomitant concentrations. (J Am Soc Mass Spectrom 1991, 2, 355-361)

$\mathrm{I}$ nductively coupled plasma mass spectrometry (ICP-MS) is a very sensitive and selective analytical tool for trace elemental analysis. Its trace level detection capabilities make it ideal for analysis of environmental and biological samples where the elements of interest are sometimes present at sub-partper-billion levels. However, the analysis of these samples is often complicated by high concomitant element concentrations. These effects have been investigated by a number of groups [1-13] with a number of trends and explanations resulting. Matrix-induced effects result from either spectroscopic interferences or nonspectroscopic interferences. Spectroscopic interferences on the analyte of interest are from ionized atomic or polyatomic species. These interferents may be singly or doubly charged ions formed from the concomitants, or polyatomic ions formed from a combination of the sample solvent, sample matrix in combination with the plasma, and/or entrained atmospheric gases. Nonspectroscopic interferences are caused by large excesses of an element or elements in the matrix that may cause plugging of the nebulizer and/or sampling cone as well as suppression or enhancement of analyte signal. This study focuses primarily on nonspectroscopic concomitant effects.

High concomitant concentrations have been reported to induce both enhancement and suppression of analyte signal, with the latter more prevalent $[1,2]$.

\footnotetext{
*Currently with the U,S. Food \& Drug Administration, National Forensic Chemistry Center, 1141 Central Parkway, Cincinnati, $O H$ 45202.

Address reprint requests to Joseph A. Caruso, Department of Chemistry, University of Cincinnati, Cincinnati, OH 45221.
}

Analyte signal suppression has been widely documented and it appears the extent of suppression is dependent upon the mass of the analyte as well as the mass of concomitant components. Heavy mass concomitant elements tend to suppress analyte ion signals more than do light mass concomitant elements, with light analyte elements being affected to a greater degree than heavy elements $[1,4,8,9]$. For example, with high $U$ matrix the $L i$ ion signal would be suppressed to a greater degree than would the $\mathrm{Pb}$ ion signal. Additionally, it has been reported that severe interferences occur in the presence of concomitant elements having low ionization potentials and therefore high degrees of ionization in the ICP [3].

One theory currently favored involves space-charge effects during the ion transmission process between the sampler and the quadrupole [4]. Gillson et al. [4] explained that interference effects can be attributed to changes in the composition of the ion beam that arise due to space-charge effects within the skimmer. Coulombic repulsion forces cause ion defocusing in high density ion beams. The degree of defocusing is dependent on the number of ions in the beam. Additionally, the instrument interface that causes sample beam expansion has an effect on the analyte ion signal. The mass of the concomitant and energy of the ions are factors in defocusing effects. The heavier analyte ions remain concentrated at the center of the beam and the lighter analyte ions are defocused more readily. This effect is most pronounced in the presence of high concomitant concentrations, with heavy mass concomitants having the greatest effect and with lighter analyte ions being affected the most. 
Several approaches to minimizing mass dependent nonspectroscopic interferences have been studied. These include the use of isotope dilution [14, 15] or standard additions [14, 15], using an internal standard $[1,8]$ or dilution [3], and flow injection sample introduction 16, 16-18]. Additionally, it has been suggested that adjusting the ion lens voltages could reduce some matrix effects $[1,4,9,13,18]$. Some of the matrix-induced analyte signal effects can be minimized through tuning the ion lenses with matrix solution (analyte plus concomitants) as opposed to tuning with a nitric acid standard solution of analyte [13].

The use of alternative plasma sources, such as mixed-gas ICPs [19-21], He ICPs [22], and microwave-induced plasmas $[23,24]$, as well as others, are being investigated for analytical applications in the detection of high ionization potential elements, such as the halogens. Recently, the He-Ar mixed-gas ICP was studied for its analytical applicability as an alternative ionization source for plasma mass spectrometry [21]. It was shown that the He-Ar plasma is capable of successfully ionizing high ionization potential elements such as $\mathrm{Cl}_{r} \mathrm{Br}$, and $\mathrm{I}_{\text {, resulting in lower }}$ limits of detection (two to three orders of magnitude) than those available from the Ar ICP. The He-Ar plasma can also be used in the determination of lower ionization potential elements.

In this study the effects of $\mathrm{Co}, \mathrm{Cd}, \mathrm{Pb}$, and synthetic ocean water (SOW) matrices on analyte signal in He-Ar mixed-gas ICP-MS are discussed. $A$ comparative study of signal optimization is discussed to examine lens tuning effects. The ion lens settings are tuned with and without the concomitant elements present for maximum ${ }^{138} \mathrm{Ba}^{+}$signal. In addition, internal standardization is used to compensate for matrixinduced effects.

\section{Experimental}

Instrumentation. The VG PlasmaQuad (VG Elemental, Winsford, Chesire, England) mass spectrometer was used in this work. Minor modifications of the instrument were made to use a mixture of $\mathrm{He}$ and $\mathrm{Ar}$ as the plasma gas. These changes have been previously discussed $[23,24]$ and include the addition of a $53 \mathrm{cfm}$ pump (single stage Edwards 80, Crawley, England) to the expansion stage to maintain pressures of $<2$ mbar (uncorrected for He-Ar mixtures). Furthermore, the gas flow system was replaced with one consisting of mass flow controllers arranged similarly to earlier systems [20, 21]. Mass flow controllers were used to control and monitor mixtures of He and Ar gas used in the outer and intermediale gas. A $20 \%$ He-Ar plasma was used throughout the study.

The ICP torch was modified to allow formation of He-Ar plasmas. These changes have been described previously and include reducing the distance between the outer and intermediate tubes [20]. This spacing was on the order of 100-150 $\mu \mathrm{m}$ and enabled the use
Table 1. Operating parameters for He-Ar ICP-MS

\begin{tabular}{|c|c|}
\hline Sampler & $\mathrm{Ni}, 0.75 \mathrm{~mm}$ orifice \\
\hline Sampling depth & $5 \mathrm{~mm}$ \\
\hline Load coil & $21 / 2$ turns \\
\hline \multicolumn{2}{|l|}{ Dimensions } \\
\hline Spray chamber & $\begin{array}{l}\text { Scott-type cooled } \\
\text { double pess, } 6^{\circ} \mathrm{C}\end{array}$ \\
\hline Solution delivery rate & $1.3 \mathrm{~mL} / \mathrm{min}$ \\
\hline \multicolumn{2}{|l|}{ Optimal operating parameters } \\
\hline Forward radiafrequency power & $1.70 \mathrm{~kW}$ \\
\hline Outer gas flow & $16 \mathrm{~L} / \mathrm{min}(20 \% \mathrm{He})$ \\
\hline Intermediate gas flow & $3.0 \mathrm{~L} / \min (2 \mathrm{O} \% \mathrm{He})$ \\
\hline Injector gas flow & $0.65 \mathrm{~L} / \mathrm{min}(0 \% \mathrm{He})$ \\
\hline \multicolumn{2}{|l|}{$\begin{array}{l}\text { Mass spectrometry } \\
\text { operating pressures* }\end{array}$} \\
\hline Expansion stage & $6.0 \times 10^{-1} \mathrm{mbar}$ \\
\hline Intermediate stage & $<10^{-4} \mathrm{mbar}$ \\
\hline Analyzer stage & $2.9 \times 10^{-6}$ mbar \\
\hline
\end{tabular}

*Pressures uncorrected for He-Ar mixtures.

of helium gas with moderate forward powers $(<2,0$ Kw). A Meinhard C-2 concentric nebulizer was used throughout the study.

In addition, the characteristics of the He-Ar mixedgas plasma necessitate modifying the load coil to change the inductance and minimize reflected power. The spacing between the coils was increased so that the net length of the load coil, from the first coil to the last coil, was $1.9 \mathrm{~cm}$ instead of $1.5 \mathrm{~cm}$. The He-Ar plasma is narrower (radially) and shorter (axially) than its Ar counterpart, thereby necessitating a decrease in the plasma sampling distance. The sampling distance was reduced to $5 \mathrm{~mm}$ from the tip of the sampler to the top of the load coil. The operating conditions for He-Ar ICP-MS are listed in Table 1.

Reagents and standards. All standard solutions (100 $\mathrm{ng} / \mathrm{mL}$ of $\mathrm{Ba}, \mathrm{Y}$, and In) were prepared by serial dilution of $1000 \mu \mathrm{g} / \mathrm{mL}$ stock solutions (Certified Atomic Absorption Standard, Fisher Scientific Co., Fairlawn, NJ) in $1 \%(\mathrm{v} / \mathrm{v})$ nitric acid. Matrix solutions containing the concomitants were prepared by dissolving salls of $\mathrm{Co}, \mathrm{Cd}$, and $\mathrm{Pb}$ nitrates, respectively, up to concentrations of $1 \%(\mathrm{w} / \mathrm{w})$ in distilled deionized (DDI) water (18 Megohm, Barnstead). Concomitant elements were chosen to study the effects of atomic mass of the concomitant element on signal suppression. The matrix solutions were spiked to conlain $100 \mathrm{ng} / \mathrm{mL}$ of $\mathrm{Ba}, \mathrm{Y}$, and In (internal standard).

A stock solution of SOW was prepared by dissolving the salts with DDI water [7]. Several SOW solutions were prepared by diluting the stock solution with DDI water to give the desired concentration of total dissolved solids, up to $0.75 \%(w / w)$, spiked to contain $100 \mathrm{ng} / \mathrm{mL} \mathrm{Ba,} \mathrm{Co,} \mathrm{In,} \mathrm{Pb,} \mathrm{and} \mathrm{Sr.}$ 
Data acquisition. All data acquisition was performed in the survey scan mode using the software (software issue 2.00) supplied with the instrument. Data acquisition conditions were as follows: mass range for $\mathrm{Co}$ concomitant was $m / z=65-200$, for $\mathrm{Pb}$ concomitant $m / z=120-204$, and for SOW concomitant $m / z=$ 43-220; 2048 channels were used and 120 sweeps per scan were used with a dwell time of $500 \mu \mathrm{s}$.

Procedures. The nonspectroscopic interferences of various sample concomitants on analyte signal were investigated. The effects of these concomitants in the He-Ar mixed-gas plasma were studied by using continuous solution nebulization. Minimization of concomitant effects was explored by using internal standardization with indium, and by optimizing ion lens potentials by tuning on the analyte of interest in the presence of the concomitant.

Experimental procedure for $\mathrm{Co}, \mathrm{Cd}, \mathrm{Pb}$, and $\mathrm{SOW}$ concomitants.

1. The ion optics were tuned for maximum ${ }^{138} \mathrm{Ba}^{+}$ signal $\left(100 \mathrm{ng} / \mathrm{mL} \mathrm{Ba}\right.$ in $1 \% \mathrm{HNO}_{3}$ ). A series of solutions, both blank and standards, were studied in the following order:

a. blank ( $\left.1 \% \mathrm{HNO}_{3}\right)$

b. standard nitric acid solution

c. $0.01 \%$ Co matrix solution (plus analyte)

Each step in the above procedure was repeated three times before advancing to the next step. To minimize carry over effects between steps, the blank solution was nebulized for $2 \mathrm{~min}$ between each step in the procedure.

2. Ion optics were tuned for maximum ${ }^{138} \mathrm{Ba}^{+}$signal in the presence of concomitant. Ion optics were tuned with each concomitant concentration and the procedure in 1 . was repeated.

The same procedure was repeated for matrix concentrations of $0.05 \%, 0.1 \%, 0.5 \%$, and $1 \%$ concomitant. This same procedure was repeated for the $\mathrm{Cd}$ and $\mathrm{Pb}$ matrices and for SOW concentrations of $0.15 \%$, $0.30 \%, 0.45 \%, 0.60 \%$, and $0.75 \%$.

The sampling cone was cleaned before advancing to a different type of matrix. Blank solutions containing the highest concentration of each concomitant were scanned and did not indicate that any analyte $(\mathrm{Ba}, \mathrm{In}, \mathrm{Y})$ impurities were present. In the following discussion standard (std) analyte refers to the analyte in a nitric acid solution and matrix analyte refers to the analyte in the presence of the concomitants. Average (avg) signals were obtained by taking the mean of three repetitions for a given solution. Additionally, analyte signals have been blank subtracted.

The normalized signal (without internal standardization) was calculated as: avg matrix analyte signal/avg std analyte signal (of that particular run)

The normalized internal standardized signals were calculated as:

(normalized analyte signal)* (avg std In signal/ avg matrix In signal)

where the analyte was normalized to the standard of that run.

\section{Results and Discussion}

The matrix effects commonly found in Ar ICP-MS are signal enhancement and/or signal suppression, and sampler clogging. Some of these effects are observed with He-Ar ICP-MS.

Effects of matrices on pressures. The pressures for the expansion and analyzer stages as well as the nebulizer were monitored throughout the study. Fluctuations in first stage pressure provide an indication of sampler cone melting or sampler cone clogging. A decrease in expansion and analyzer stage pressures indicates blockage of the sampler cone, and a clogged nebulizer is indicated by an increase in nebulizer gas pressure. Unlike Ar ICP-MS [13], solutions up to $1 \%$ matrix were nebulized without any significant changes to the expansion stage pressure or nebulizer pressure, with one exception. The nebulizer pressure increased with the $1 \%$ Co matrix solution during the tuning process. The normal wash cycle was sufficient to bring the pressure back to the original value. There were no visible signs of salt deposition on the sampler in any of the studies.

Effects of matrices on ion lens voltages. Ion optics are used to focus ions prior to entering the mass spectrometer. The VG PlasmaQuad, used in this study, has a series of nine lenses with variable voltages that can be adjusted for maximum ion signal (Figure 1). The extractor lens extracts positive ions and directs them toward the lens stack. The ions are entrained by the collector electrode. Lenses L1 and L2 focus ions through the differential pumping aperture (DA). The DA focuses ions from the intermediate stage to the analyzer stage. The emerging ions are refocused to the quadrupole by $\mathrm{L} 3$ and $\mathrm{L} 4$. Potential ranges for the lenses are listed in Table 2.

In this study, all of the ion lenses were adjusted for maximum ion signal of ${ }^{138} \mathrm{Ba}^{+}$in either a standard nitric acid solution or in the matrix solution. Barium was chosen because it is a mid mass element. Changes in lens voltage were noted for each matrix concentration. The extractor, collector, and differential aperture were affected most when the lenses were tuned in the presence of $\mathrm{Pb}, \mathrm{Cd}$, and $\mathrm{Co}$ matrix solutions. Generally, these lenses are the most sensitive of the series in plasma mass spectrometry with the VG PlasmaQuad. The collector and extractor had to be 


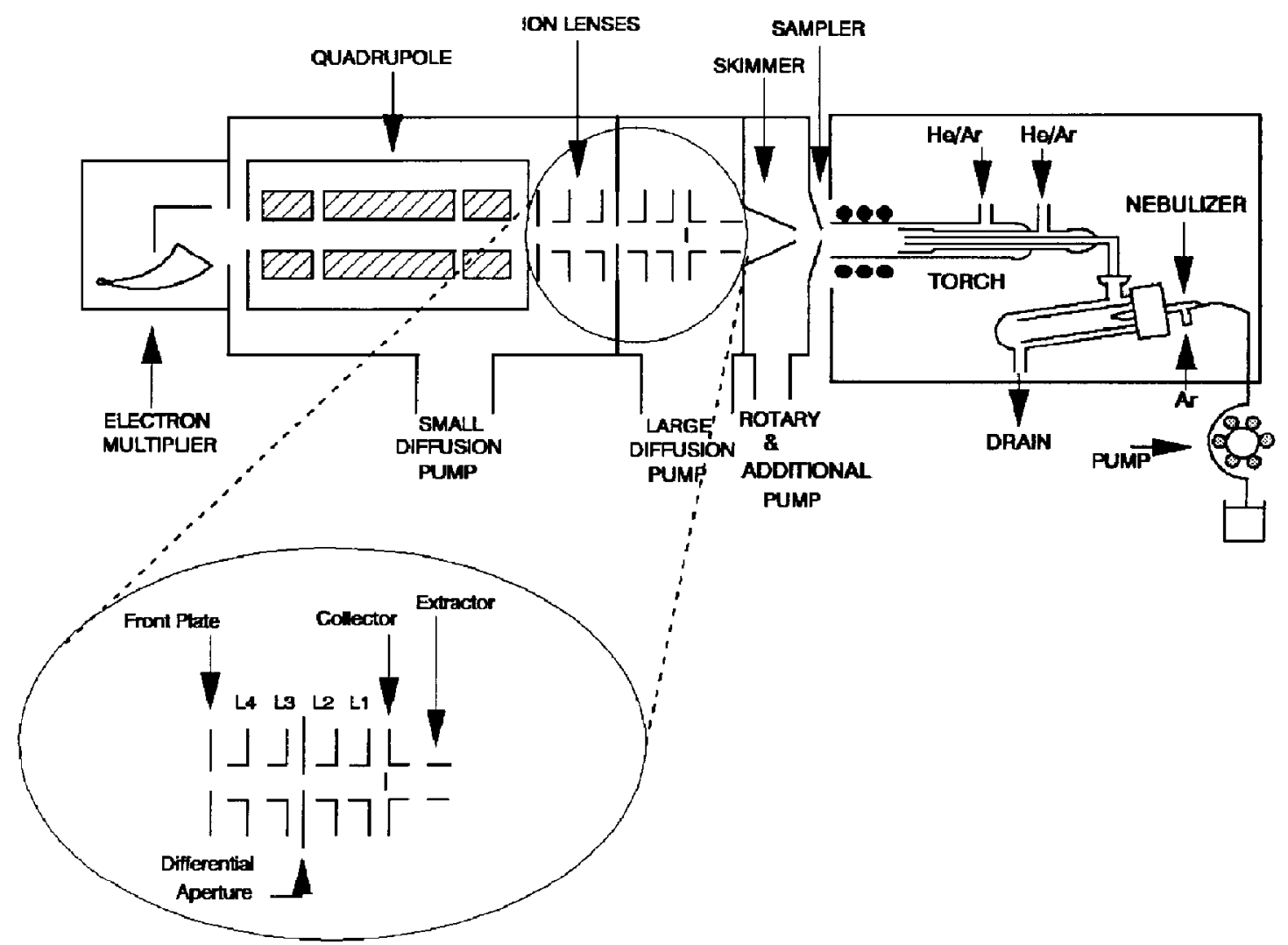

Figure 1. Experimental setup for $\mathrm{He-Ar}$ ICP-MS.

increased to higher negative potentials while the differential aperture increased to more negative potentials for $\mathrm{Pb}$ but toward positive potentials for $\mathrm{Cd}$ and $C o$. Ion lenses retuned on the standard solution between each matrix showed somewhat different results. The voltage on the collector varied only slightly, whereas the extractor, L2, and DA fluctuated up and down. The front plate setting increased considerably for Cd, -100 volts $(0.01 \%)$ to -25 volts $(1 \%)$. The variation in ion lens settings for the analyte signal in the $\mathrm{Pb}$ matrix study tuned either with a standard solution or in the presence of the concomitant ele-

Table 2. Ion lens potential range

\begin{tabular}{lcc}
\hline & \multicolumn{2}{c}{ Voltage } \\
\cline { 2 - 3 } Lens & Dial 0.00 & Dial 10.00 \\
\hline \hline Extractor & -100 & -1000 \\
Collector & -150 & +150 \\
Lens 1 & -150 & +150 \\
Lens 2 & -150 & +150 \\
Lens 3 & -250 & +250 \\
Lens 4 & -250 & +250 \\
Differential aperture & -150 & +150 \\
Quadrupole front plate & -150 & +150 \\
Pole bias & -30 & +30 \\
\hline
\end{tabular}

ment is shown in Figure 2. The concentrations in the tuned on the standard study represent the concentration of the matrix solution to be analyzed after tuning. Lens dial settings indicate lens potential (see Table 2).

Ion lens voltage values for the lowest and highest percent matrix for $\mathrm{Pb}, \mathrm{Cd}$, and $\mathrm{Co}$ are listed in Table 3 . The purpose of the ion lenses is to direct positive ions to the quadrupole, so it is expected that a more negative potential is needed as the concentration of positive ions increases. Lens potentials should remain relatively constant when tuning on a standard nitric acid solution, as is seen in this study. Differences in potentials are probably due to the ion lenses becoming coated as the matrix ion flow increases. When tuning in the presence of the matrix there are additional positive ions present, and it was observed that as the concentration of the matrix increases so did the potential on the lenses. Additionally, the space-charge effect theory suggests that the analyte ions will become increasingly defocused as the concentration of the concomitant element increases. A larger potential will be needed to refocus the analyte ions. However, a point may be reached at which a plateau occurs and additional concomitant elements will not affect the signal to any appreciable degree [8].

In this study it was observed that lens potential variation was minimal when the analyte ion signal 


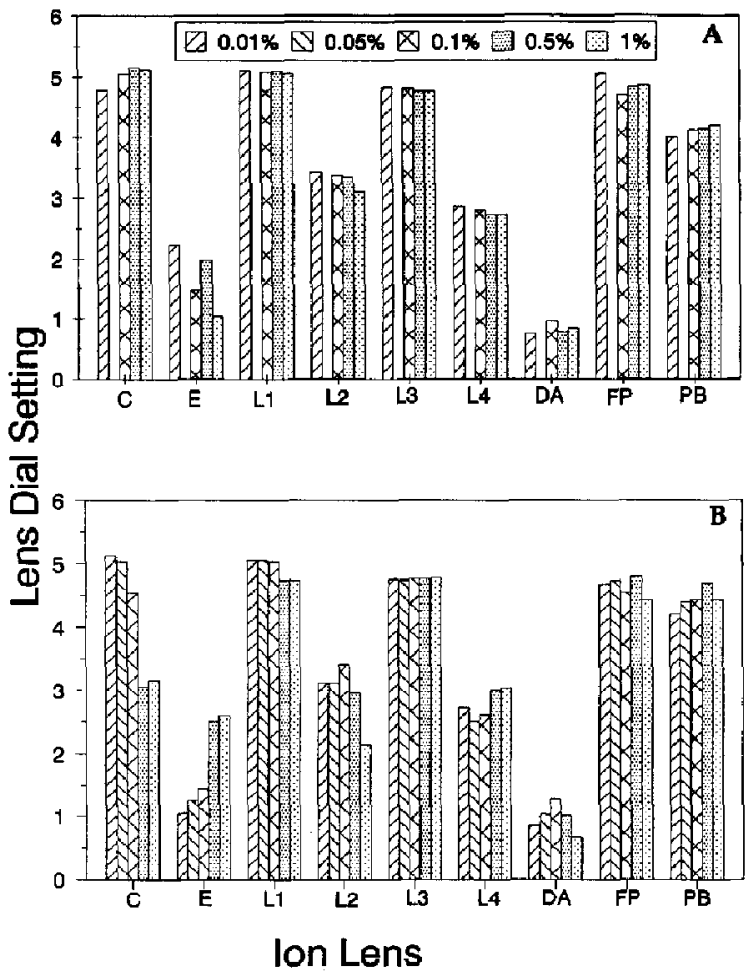

Figure 2, Effects of various $\mathrm{Pb}$ matrix concentrations (indicated in inset) on ion lens potentials. Signal was maximized for $\mathrm{Ba}$ $(m / z=138)$. (a) Tuned on standard nitric acid solution. (b) Tuned in the presence of matrix.

was optimized on a standard nitric acid solution, as shown in Figure 2a. However, the extractor lens shows somewhat different results in that there is a considerable difference in voltage between the $0.01 \%$ matrix $(-325 \mathrm{v})$ and the $1 \%$ matrix $(-200 \mathrm{v})$. Analyte ion signal optimization conducted in the presence of the matrix showed different results. As the concomitant element concentration increased the voltage required for maximum ion signal also became more negative. The largest variations in potential, for the $\mathrm{Cd}$ and $\mathrm{Co}$ matrices, occurred between the $0.01 \%$ and $0.05 \%$ matrix solutions. The heavier mass $\mathrm{Pb}$ matrix showed

Table 3. Ion lens potentials (V)*

\begin{tabular}{lrrrrrrrr}
\hline \multirow{2}{*}{$\begin{array}{c}\text { Percent } \\
\text { matrix }\end{array}$} & \multicolumn{2}{c}{$\mathrm{Pb}$} & & \multicolumn{2}{c}{$\mathrm{Cd}$} & & \multicolumn{2}{c}{$\mathrm{Co}$} \\
\cline { 2 - 3 } \cline { 7 - 8 } & $0.01 \%$ & $1 \%$ & & $0.01 \%$ & $0.05 \%$ & & $0.01 \%$ & $0.05 \%$ \\
\hline \hline Extractor & -325 & -200 & -130 & -120 & -120 & -125 \\
& -200 & -400 & -120 & -300 & -125 & -200 \\
Collector & -30 & -20 & -25 & -25 & -25 & -25 \\
& -20 & -75 & -25 & -50 & -20 & -30 \\
Differential & -130 & -120 & -105 & -130 & -150 & -150 \\
Aperture & -120 & -135 & -145 & -140 & -150 & -140 \\
\hline
\end{tabular}

aFirst row for each lens contains values when tuned on the nitric acid standard. Second raw contains values when tuned on the matrix. significant ion lens potential differences throughout the matrix concentration range, as shown in Figure $2 \mathrm{~b}$. The heavy mass matrix causes more defocusing of ${ }^{138} \mathrm{Ba}^{+}$analyte ions, requiring larger negative lens potentials to refocus the ions. This is especially so when high concentrations of heavy mass matrices are present. This observation is similar to results observed for argon plasma ICP-MS [4]. Ion trajectories are a function of ion kinetic energy, which is mass dependent. The heavier mass lead matrix ions may maintain their trajectories more so than the lighter mass matrix ions and thus interfere to a greater extent with optimal transmission of barium ions.

Effects of retuning on the ion signal. As stated previously the ion optics were retuned for ${ }^{138} \mathrm{Ba}^{+}$in each matrix solution. For the lead and cobalt matrix the maximum ion signal steadily decreased with increasing matrix concentration $(0.01 \%$ to $1 \%)$. In the $\mathrm{Pb}$ matrix the Ba signal decreased by a factor of 10 from the $0.10 \%(620,000$ counts per second) matrix to the $1 \%(53,000 \mathrm{cps})$ matrix. The Ba ion signal decreased from 580,000 cps in $0.01 \%$ matrix to $21,000 \mathrm{cps}$ in $1 \%$ matrix with the cobalt matrix, and decreased from $590,000 \mathrm{cps}$ in $0.01 \% \mathrm{Cd}$ matrix to $172,000 \mathrm{cps}$ in $1 \%$ $\mathrm{Cd}$ matrix. In the SOW matrices the ion signal was constant until $0.75 \%$ SOW, where it then decreased from 410,000 cps to 172,000 cps. The above discussion is supported by Figure 3.

In addition to tuning on elements in the presence of the matrix, a study was conducted in which the ion optics were retuned on a nitric acid standard solution. The signal intensity for this solution was noted before any retuning was conducted to determine if conditions for optimal barium ion signal had changed. The $\mathrm{Pb}$ matrix showed the largest change in barium ion signal intensity between the lowest matrix concentrations $(0.01 \%$ and $0.05 \%)$. The ion signal resulting from the standard barium solution decreased from 522,000 cps before introducing the $0.01 \%$ matrix solution to

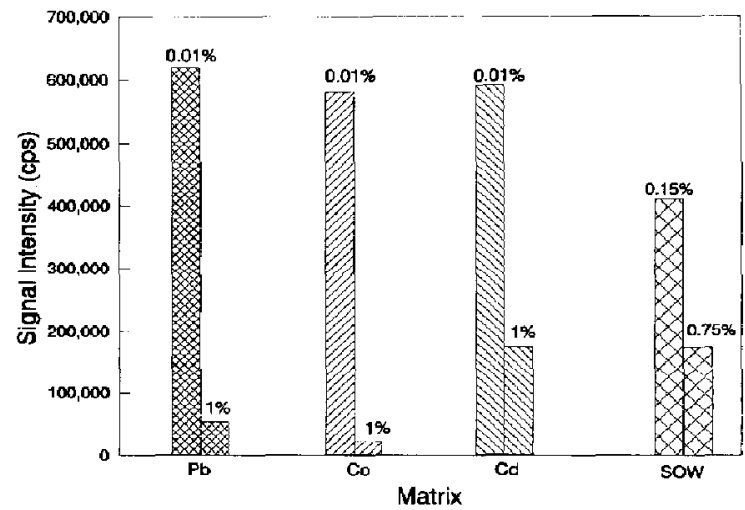

Figure 3. Effects of concomitant element concentration on ${ }^{138} \mathrm{Ba}^{+}$signal. Ion lenses tuned in the presence of concomitant elements. 

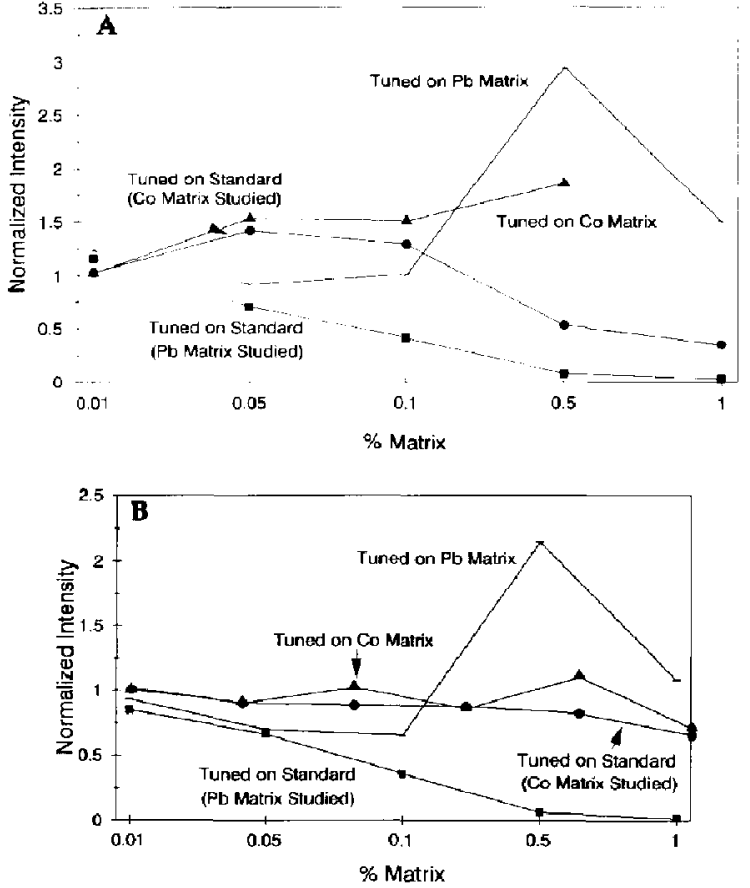

Figure 4. (a) Yttrium signal in the presence of $\mathrm{Co}$ and $\mathrm{Pb}$ matrices. (b) Barium signal in the presence of $\mathrm{Co}$ and $\mathrm{Pb}$ matrices. The ion lenses were tuned on ${ }^{138} \mathrm{Ba}^{1}$ both in the presence and absence of matrix. Normalized intensity calculated by using eq 1 .

130,000 cps immediately following. Upon retuning the ion lenses with the standard solution, the signal increased to 729,000 cps. Less dramatic effects were observed with $\mathrm{Cd}$ and $\mathrm{Co}$ matrix solutions, where the largest difference in barium ion signal intensity with the Co matrix was a $27 \%$ decrease between $0.01 \%$ and $1 \%$ matrix solutions; retuning improved the signal by $3 \%$ at most. The $\mathrm{Cd}$ matrix showed similar results with only a $9 \%$ difference between the highest and lowest maximum ion counts, and retuning improved the standard signal $12 \%$ at most. The greatest effects were seen in the heavy mass $\mathrm{Pb}$ matrix and less so with the light mass Co matrix.

As seen above, barium analyte ion signal can change drastically by retuning the lenses. In fact, the magnitude of analyte signal intensity is dependent upon the tuning procedure used. The differences in ion signal for $\mathrm{Y}^{\mathrm{in}} \mathrm{CO}$ and $\mathrm{Pb}$ matrices are shown in Figure 4a. Note the large difference in ion signal for $0.5 \% \mathrm{~Pb}$ matrix when tuned on the standard solution showing signal suppression as compared to tuning on the matrix, which shows signal enhancement. The presence of a large amount of matrix seriously affects ion lens tuning in He-Ar ICP-MS. The $\mathrm{Pb}$ matrix was the first matrix solution to be analyzed in the series and showed the most significant variations in analyte signal and required lens settings. Although the lenses were retuned on a standard solution throughout, similar results were observed for both $\mathrm{Ba}$ and In analytes.

In Ar ICP-MS it has been reported that lighter elements are affected most by the presence of highly concentrated matrices and that heavy mass matrices cause the largest signal deviations $[1,4,8,9]$. This trend is also found in the He-Ar plasma when the lenses are tuned in the presence of concomitant elements such as lead and cobalt, as shown in Figure 4. The data in Figure 4 indicate that $Y$ analyte is affected more by the heavy $\mathrm{Pb}$ matrix $(m / z 208)$ than by $\mathrm{Co}$ $(m / z$ 59). Similar observations have been reported for the Ar ICP $[1,4,8$, 9]. Barium is affected by high matrix concentrations in a manner similar to yttrium (see Figure $4 b)$. However, yttrium $(m / z 89)$ is a lighter element than $\mathrm{Ba}(m / z 138)$ and, as expected, is influenced more. Heavier mass matrices cause more matrix-induced effects. Yttrium shows more enhancement than does $\mathrm{Ba}$ in the $\mathrm{Pb}$ matrix.

Internal standardization. The use of internal standards has been suggested as a possible means of compensation for matrix-induced analyte signal problems [8]. Indium $(m / z .115)$ was chosen as an internal standard in this study because it is a mid-mass element. Internal standard signals were calculated by using eq 2 . The results of adjusting the analyte ion signal with indium in $\mathrm{Pb}$ and $\mathrm{Co}$ matrices are shown in Figures 5
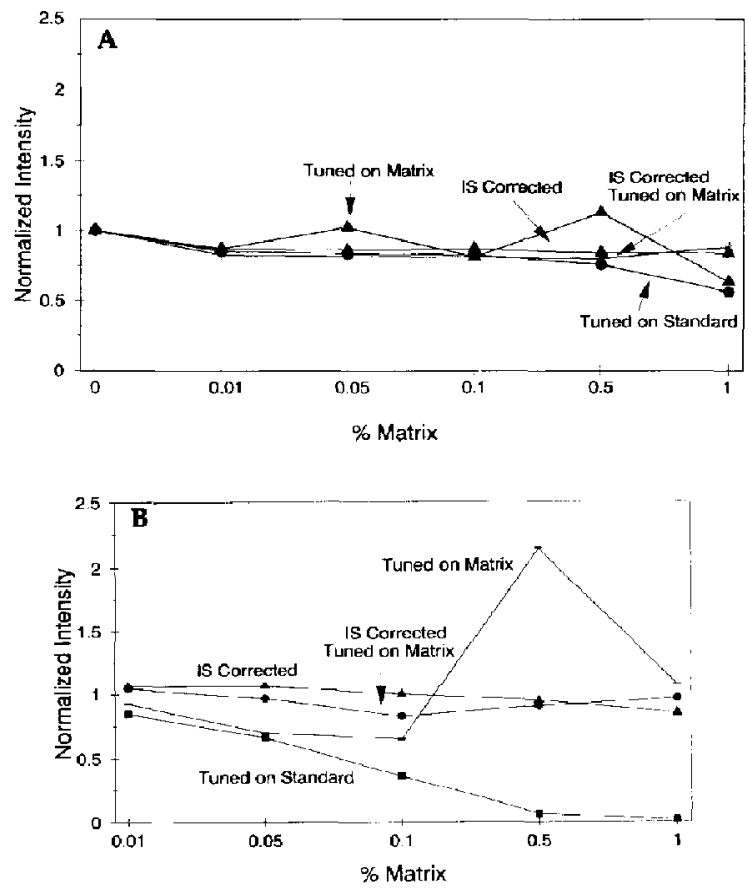

Figure 5. Comparison of ${ }^{138} \mathrm{Ba}$ ion signal in (a) Co matrix and (b) $\mathrm{Pb}$ matrix: tuned on the matrix, tuned on the standard, and with internal standard (IS) correction. Normalized intensity calculated by using eqs 1 and 2 . 

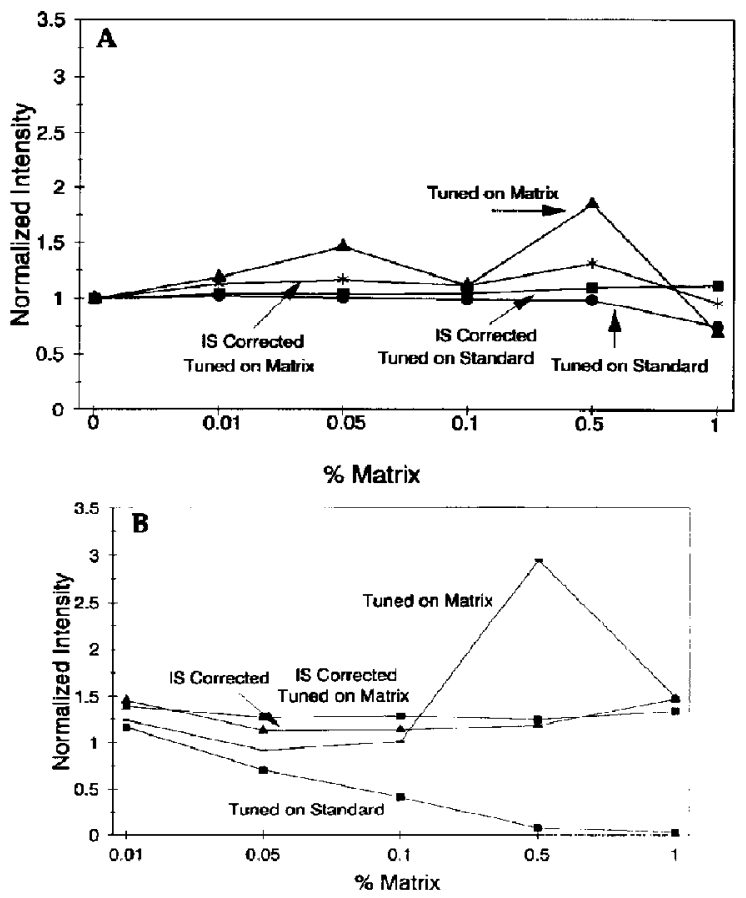

Figure 6. Comparison of ${ }^{89} \mathrm{Y}$ ion signal in (a) Co matrix and (b) Pb matrix: tuned on the matrix, tuned on the standard, and with internal standard correction (IS Corrected). Normalized intensity calculated by using eqs 1 and 2 .

and 6. Compensation for matrix-induced effects is successful when the normalized signal is equivalent to unity. The Co matrix-induced effects on the barium jon signal for both types of tuning along with the effectiveness of internal standardization procedures are shown in Figure 5a. The effects of the $\mathrm{Pb}$ matrix on the barium ion signal and internal standard correction for both tuning procedures are shown in Figure $5 b$. Notice how the normalized barium ion signal approaches unity for both tuning cases and in both the $\mathrm{Co}$ and $\mathrm{Pb}$ matrices. Similar results for yttrium in cobalt and lead matrices are shown in Figure 6. Cadmium matrix solutions study yielded results similar to those of the Co study. The SUW matrix, which is composed of lower mass elements, had results similar to those found in the $\mathrm{Co}$ and $\mathrm{Cd}$ matrices.

\section{Conclusions}

Matrix effects are encountered in He-AR ICP-MS. Unlike the Ar-ICP, He-Ar ICP-MS is not as subject to sampler clogging at high matrix concentrations (up to $1 \%)$. Additionally, both analyte ion signal suppression and enhancement can be observed depending on the matrix and ion lens tuning procedure. In a similar study with Ar ICP-MS [13], high matrix concentrations resulted in analyte suppression. Tuning on the matrix causes enhancement at $0.5 \%$, while tuning on the standard solution causes signal suppression. Additionally, lighter elements, such as yttrium undergo more matrix-induced variation than do heavy elements and are affected more by a heavy element matrix. Matrix effects can be compensated for through the use internal standardization with elements such as indium.

\section{Acknowledgments}

The authors are grateful to the National Institute of Environmental Health Sciences for support of this study under grant nos. ES 03221 and ES 04908 . We are also thankful to the NIH-BRS Shared Instrument Grant Program for providing the VG PlasmaQuad through grant no. SIORR02714.

\section{References}

1. Beauchemin, D.; McLaren, J. W.; Berman, S. S. Spectrochim. Acta 1987, 42B, 467.

2. Gregoire, D. C. Spectrochim. Acta 1987, 42B, 895.

3. Tan, S. H.; Horlick, G. J. Anal. At. Spectrom. 1987, 2, 745.

4. Gillson, G. R,; Douglas, D. J.; Fulford, J. E.; Halligan, K. W.; Tanner, 5. D. Anal. Chem. 1988, 60, 1472.

5. Vandecasteele, C.; Nagels, M.; Vanhoe, H.; Dams, R. Anal. Chim. Acta 1988, 211, 91.

6. Hutton, R. C.; Eaton, A. N. J. Anal. At. Spectrom. 1988, 3, 547.

7. Brotherton, T. J.; Shen, W. L.; Caruso, J. A. I. Anal. At. Spectrom 1989, 4, 39.

8. Thompson, J. J.; Houk, R. S. Appl. Spectrosc. 1987, 41, 801.

9. Crain, J. S.; Houk, R. S.; Smith, F. G. Spectrochim. Acta 1988, 43B, 1355.

10. Olivares, J. A.; Huuk, R. S. And. Chem. 1986, 58, 20.

11. Gregoire, D. C. Appl. Spectrosc. 1987, 41, 897.

12. Satzger, R. D. Anal. Chem. 1988, 60, 2500.

13. Wang, J.; Shen, W. L.; Sheppard, B. S.; Evans, E. H.; Fricke, F. L.; Caruso, J. A. J. Anal. At. Spectrom. 1990, 5, 445.

14. McLaren, J. W.; Beauchemin, D.; Berman, S. S. Anal. Chem. 1987, 59, 610.

15. Garbarino, J. R.; Taylor, H. E. Anal. Chem. 1987, 59, 1568.

16. Thompson, J. J.; Houk, R. S. Anal. Chem. 1986, 58, 2541.

17. Dean, J. R.; Ebdon, L.; Crews, H. M.; Massey, R. C. I. Anal. At. Spectrom. 1988, 3, 349.

18. Vickers, G. H.; Ross, B. S.; Hieftje, G. H. Appl. Spectrosc. 1989. 2, 1330.

19. Choot, E. H.; Horlick, G. Spectrochim. Acta 1986, 41B, 906.

20. Sheppard, B. S.; Caruso, J. A.; Wolnik, K. A.; Fricke, F. L. Appl. Spectrosc. 1990, 44, 712.

21. Sheppard, B. S.; Shen, W. L.; Davidson, T. M.; Caruso, J. A. J. Anal. At. Spectrom. 1990, 5, 697.

22. Chan, S.; Montaser, A. Spectrochim. Acta 1985, 40B, 1467.

23. Brown, P. G.; Davidson, T. M.; Caruso, J. A. I. Anal. At. Spectrom. 1988, 3, 763.

24. Creed, J. T,; Mohamad, A. H.; Davidson, T. M.; Ataman, G.; Caruso, J. A. J. Anal. At. Spectrom. 1988, 3, 923. 